\title{
Anterior Controllable Antedisplacement and Fusion (ACAF) technique for the treatment of multilevel cervical spondylotic myelopathy with spinal stenosis (MCSMSS): a retrospective study of $\mathbf{4 5}$ cases
}

Xi Luo

Changzheng Hospital

Kaiqiang Sun

Changzheng Hospital

Jingchuan Sun

Changzheng Hospital

Shunmin Wang

Changzheng Hospital

Yuan Wang

Changzheng Hospital

Haisong Yang

Changzheng Hospital

Jialin Jiang

Changzheng Hospital

Yongfei Guo

Changzheng Hospital

Jiangang Shi ( $\sim$ brookluo@outlook.com )

Changzheng Hospital

Research article

Keywords: cervical spondylotic myelopathy; spinal stenosis; multilevel; anterior controllable antedisplacement fusion (ACAF)

Posted Date: August 9th, 2019

DOl: https://doi.org/10.21203/rs.2.12543/v1 
License: (c) (i) This work is licensed under a Creative Commons Attribution 4.0 International License. Read Full License

Version of Record: A version of this preprint was published at Clinical Spine Surgery: A Spine Publication on March 17th, 2021. See the published version at https://doi.org/10.1097/BSD.0000000000001144. 


\section{Abstract}

Background To investigate the clinical effect of anterior controllable antedisplacement and fusion (ACAF) technique for the treatment of multilevel cervical spondylotic myelopathy with spinal stenosis (MCSMSS), and compare ACAF with hybrid decompression fixation (HDF). Methods A retrospective analysis of 85 cases with MCSMSS was carried out. 45 patients were treated with ACAF, while 40 patients were treated with HDF. The operation time, intraoperative bleeding volume, postoperative complications, Japanese Orthopaedic Association (JOA) score, Neck Disability Index (NDI) score, Computed Tomography (CT) transverse measurement, cervical curvature and Kang's grade were compared between two groups. Results The patients were followed up for 12 to 17 months. Compared with HDF, ACAF group achieved better decompression according to CT measurement and Kang's grade $(P<0.05)$, and recovered to a greater cervical Cobb's angle $(P<0.05)$. However, JOA score and NDI index showed no significant difference one year after surgery $(\mathrm{P} \otimes 0.05)$. Additionally, ACAF presented longer operation time and greater intraoperative blood loss $(P<0.05)$. As to complications, ACAF developed less incidences of cerebrospinal fluid examination (CSF) leakage, neurologic deterioration, epidural hematoma and C5 palsy by comparing with HDF. Conclusions ACAF is an effective method for the treatment of MCSMSS. Compared with HDF, ACAF has the advantages of significant decompression, increasing cervical curvature, and reducing the incidences of complications.

\section{Background}

Multilevel cervical spondylotic myelopathy with spinal stenosis (MCSMSS) is a disorder of spinal cord dysfunction characterized by involving three or more cervical segments, which is caused by congenital, developmental or degenerative factors which results in spinal stenosis and compression of spinal cord and its blood vessel at the levels of both intervertebral disc and vertebra[1]. Surgery plays a vital treatment part because of the progressive feature of MCSMSS, especially for patients with intolerable symptoms and suspected neurological damage[2], but considering the multilevel and severe compression, the choice of surgery is controversial[3]. For single-level compression, anterior cervical discectomy and fusion (ACDF) is the "gold standard" for surgical treatment $[4,5]$. However, it is difficult for ACDF to achieve complete compression when faced with multilevel lesions. As to the anterior cervical corpectomy decompression and fusion (ACCF), although its effect of decompression is significant, the instability of cervical spine and the high risk of complications cannot be ignored[6].

In order to better solve the clinical problems of MCSMSS, we proposed to utilize a novel treatment scheme of anterior controllable antedisplacement and fusion (ACAF) on MCSMSS. ACAF is characterized by hoisting the anterior wall of the spinal canal to make it move forward, thus expanding the volume of the spinal canal and relieving the compression. The purpose of this study was to compare ACAF with hybrid decompression fixation (HDF, the combination of ACDF and ACCF) in treatment of MCSMSS[7], and to briefly introduce ACAF surgical technique, which are reported as follows.

\section{Methods}




\subsection{General information}

From January 2017 to January 2019, 85 patients diagnosed with MCSMSS and treated with ACAF (45 cases) or HDF (40 cases) were selected for the study. X-ray, Computed Tomography (CT) and Magnetic Resonance Imaging (MRI) of cervical spine were taken before operation. Selection criteria: (1) Confirmed by cervical spinal stenosis (the sagittal diameter of the spinal canal is less than $12 \mathrm{~mm}$ ), and the conservative treatment is ineffective; (2) cervical spondylotic myelopathy involving segments $\geq 3$. Exclusive criteria: (1) deformity, ankylosing spondylitis, rheumatoid arthritis and other diseases involving the cervical spine; (2) cervical spine trauma, surgical history; (3) severe osteoporosis.

All patients agreed to the record of research data, and signed the informed consent.

\subsection{Operative methods}

ACAF group: (1) After general anesthesia, supine position was taken, cervical oblique incision was made through the anterior right side to expose deep structures. (2) The responsible intervertebral disc, anterior and posterior edge osteophyte were removed until the posterior longitudinal ligament (PLL) was exposed. Then, at the cephalic and caudal ends of the planned hoisting segments, the PLL should be bitten in intervertebral space, while the rest of PLL need not be deal with. (3) According to the requirement for decompression, the certain thickness of anterior part of the vertebra was removed, and the space was reserved for the responsible segment to be hoisted forward. (4) According to the decompression width needed, the grooving position was selected on the anterior surface of vertebra, where was usually the inner edge of Luschka's joint. Grinding drill was used to dig deep along the grooving lines on both sides, and the bone was chisel away to reach the cortex of the posterior wall of the vertebra. The posterior wall of the vertebra was bitten on one side. The other side was temporarily retained to maintain the stability of the vertebra. (5) Intervertebral fusion cages, pre-curved titanium plate and vertebral screws were installed, and then the posterior wall of the vertebra on the other side was resected to make the vertebra free. (6) The titanium plate was pulled forward. At this time, vertebrae and compression mater would move forward together until the vertebrae and the titanium plate were closely joined. Finally, the incision was flushed, hemostasis and drainage were performed, and suture was finished layer by layer. The diagram of the procedure of ACAF is presented as Fig 1.

HDF group: ACCF was performed at the segments with severe compression, and ACDF was performed at other segments. The specific steps are as follows:

(1) ACCF: Exposure process was the same as ACAF group.

Degenerative discs and osteophytes in the intervertebral space was removed, until reaching hook joints on both sides. Subtotal resection of adjacent vertebrae was performed, and osteophytes were removed. Titanium cages filled with broken bones were placed after decompression. Finally, titanium plate and screw were installed. 
(2) ACDF: Other processes were the same as ACCF. After discectomy, the osteophytes at posterior margin of the vertebra were removed, and the intervertebral space was expanded by distractor to normal height. Intervertebral fusion cage was selected and inserted into the intervertebral space. Titanium plate and screw fixation was finally performed.

Postoperative management was as ACAF's.

All operations were performed by surgeons of the same team. Negative pressure drainage tubes were placed and pulled out 24 to 48 hours after operation. All patients were fixed with external cervical bracket for 3 months.

\subsection{Observation Indicators}

\subsubsection{General indicators}

Age, sex, operative levels, operation time, and intraoperative bleeding volume and complications were recorded.

\subsubsection{Functional evaluation}

Before and 1 year after operation, neurological function was evaluated by Japanese Orthopaedic Association (JOA) score and Neck Disability Index (NDI) score.

\subsubsection{CT transverse measurement}

By utilizing the measuring tools embedded in software of picture archiving system, 3 parameters were measured on CT axis images to evaluate the effects of decompression. The definitions of the parameters were as follows:

(1) Transverse area of spinal canal: the area surrounded by the posterior edge of vertebra (in ACAF) or the titanium cage (in HDF), the inner side of vertebral plate and the inner side of pedicle.

(2) Decompression width: the distance between the double sides grooves at the anterior wall of spinal canal

(3) Sagittal diameter of spinal canal: the distance between the posterior edge of vertebra or the titanium cage, and the base of spinous process

The data was from all the operative segments, and were measured at the middle level of vertebra. The measuring diagram is depicted as Fig 2.

\subsection{4. $\mathrm{C}_{2-7}$ Cobb's angle}

The a Cobb's angle method was used to evaluate cervical curvature on the lateral images of X-ray, and the angle is formed by vertical lines of the upper edge of $\mathrm{C}_{2}$ and the lower edge of $\mathrm{C}_{7}$. 


\subsubsection{Kang's grade}

Kang's MRI grading system was used to assess the degree of cervical spinal cord compression[8, 9], and the specific criteria is : grade-0, no spinal canal stenosis; grade-1, subarachnoid compression exceeded 50®; grade-2, spinal cord compression deformed; grade-3, spinal cord T2 weighted signal changes.

All the parameters were measured by two senior spine surgeons independently, and the average value of each parameter at each level was taken for independent calculation.

\subsection{Statistical methods}

SPSS 22.0 was used for statistical analysis. Measurement data were expressed as mean \pm standard deviation ( ). Paired t test was used for intragroup comparison. Two independent samples t-test for intergroup comparison, Chi-square test was for the categorical data comparison. Test level was $\mathrm{a}=0.05$.

\section{Results}

\subsection{Demographics}

All the enrolled 85 cases completed the operation successfully, with clinical symptoms relieved and spinal cord function improved, and no incision infection and nerve injury occurred. 85 cases were followed up for 12 to 17 months, with an average of 15.6 months.

Among 85 cases, there were 45 cases in the ACAF group, including 33 males and 12 females, aged 40-72 (mean, 53.0+10.1) years; 40 cases in the control group, including 22 males and 18 females, aged 38-75 (mean, 56.1+9.1) years. There was no significant difference in gender, age and operative levels between the two groups $(P>0.05)$. The general data is shown in Table 1 .

\subsection{CT measuring}

The preoperative diameter of spinal canal, decompression width and spinal canal area in two groups showed no significant differences ( $P \otimes 0.05)$. After surgery, compared with HDF group, the ACAF group presented a better decompression at C3, C4, C5, and C6 levels ( $P \otimes 0.05)$. The data is shown in Table 2.

\subsection{Clinical outcomes}

According to the data, there was no significant difference in preoperative JOA score, NDI index, Cobb's angle and Kang's grade between the two groups $(P>0.05)$. One year after operation, both groups were significantly improved in terms of JOA score and NDI index $(P<0.05)$. However, there was no significant difference in JOA score and NDI index between two groups $(P>0.05)$. As to postoperative Cobb's angle and Kang's grade, ACAF revealed its unique superiority, with higher Cobb's angle ( $P \otimes 0.05)$, and lower Kang's grade ( $P \otimes 0.05)$, comparing to HDF. Additionally, the result showed a higher cost in operative time and intraoperative bleeding in ACAF group ( $P \otimes 0.05)$. 
As to the complications, 5 patients (12.5区) developed cerebrospinal fluid examination (CSF) leakage, 2 patients (5.08) had neurologic deterioration, 2 patients (5.08) presented with epidural hematoma, 2 patients (5.0区) suffered from C5 palsy, and 3 (7.5区) developed dysphagia in HDF group. Additionally, at the final follow-up, and 4 patients (10.0囚) presented with implant-related complications in HDF group, including 2 cases of cage subsidence and 2 cases of delayed union. In contrast, ACAF group demonstrated only 1 case (2.2区) of CSF leakage and 4 cases (8.8区) of dysphagia, without other complications.

The data is shown in Table 1 and 3.

\subsection{Explanatory cases}

Case 1. Complained of numbness of both arm with gait disturbance for 3 years. The numbness had been aggravated 2 months before presentation. ACAF was performed at $\mathrm{C} 4$ and $\mathrm{C} 5$. After the operation, the patient showed notable relief of neurologic function. Enough decompression of the spinal cord was achieved according to imaging. The JOA score increased from 7 to 11. There were no complications during follow-up. The imaging examination is shown in Fig 3.

Case 2. Presented with spastic weakness of both hands and gait instability for 2 years. The symptoms progressively worsen in 3 months before presentation. ACDF was performed at C4-5 level, and ACCF at C5-6 level. After the operation, the patient's myelopathy showed marked recovery. Preoperative and postoperative JOA score was 8 and 12, respectively. No complications were observed during follow-up. The imaging examination is shown in Fig 4.

\section{Discussion}

\subsection{Technical features of HDF}

The purpose of HDF is to use ACDF in slightly diseased segments and ACCF in the severe[10], so as to preserve the vertebrae, and relieve the compression as much as possible. Compared with ACCF, HDF is associated with less blood loss, less complications, and higher fusion rate[4, 11]. However, when involving the multilevel compression, in order to avoid dural tear and spinal cord injury, it is sometimes difficult to take full advantages of HDF and complete the thorough compression[12], and residual compressive matter will still produce compression[13]. At the same time, the risk of complications such as dural tear, spinal cord injury and titanium cage sinking still cannot be ignored in HDF just like in ACCF[14].

\subsection{Technical features of ACAF}

In ACAF, vertebrae-compression complex (VCC) is a vital concept that includes vertebrae of responsible segments, osteophytes, proliferative and ossified PLL, which is freed out through grooving at both sides of vertebrae, and it can be regarded as the anterior wall of the spinal canal. By moving VCC forward can surgeons expand the volume of the spinal canal and retain more bone of vertebrae simultaneously. 
During the operation, the forward movement of VCC can be monitored by X-ray, so that the whole process of hoisting and spinal cord antedisplacement can be controlled, and the "anatomical reduction" of spinal cord can be achieved. On the other hand, according to experience, the grooving position is roughly at the inner edge of Luschka's Joint, which not only ensures the enough decompression width (the effective range almost reaches the medial wall of the pedicle), but also avoids the injury of vertebral artery. Compared with the HDF operated in the anterior median region of cervical spine, ACAF operation mainly focuses on the region around Luschka's joint, which is far away from spinal cord and its artery traveling area, constructing a "safe space" for spinal cord and reduces the risk of spinal cord injury.

\subsection{Advantages of ACAF in the treatment of MCSMSS}

(1) Good recovery of spinal cord morphology and CSF space

It is observed that the Kang's grade of ACAF decreased significantly, which means the compression of CSF band and spinal cord is confirmed on MRI. By pulling the VCC, spinal cord can not only restore normal anatomical morphology, but also avoid traction caused by backward drift, and the recovery of CSF space is also beneficial to the improvement of symptoms after surgery $[15,16]$.

\section{(2) Fewer complications}

1) C5 palsy

Due to the technical limitations of ACCF, the true decompression width of spinal canal is significantly smaller than the slotting width of the anterior vertebral surface, resulting in incomplete decompression at the outlet of the nerve root[17]. However, in ACAF, the width of decompression is fully guaranteed by vertical grooving and vertebral hoisting. Anatomically, the Luschka's joint participates in the formation of nerve root outlet. So, when VCC moves forward, the space around the outlet of nerve root will be greatly expanded to achieve nerve decompression, meanwhile, the risk of nerve root traction is further avoided due to the recovery of curvature and position of spinal cord.

2) Postoperative hematoma and CSF leakage

When venous plexus and dura mater adhere to surrounding ossified mater, operation is easy likely to cause hematoma and CSF leakage[18]. In ACAF, the operation does not directly involve the dura mater and intraspinal venous plexus. When there exists adhesion between dura and ossified mater, after VCC is moved forward, these structures are still in previous connection relationship, which reduces the risk of dura tear and hematoma. Furthermore, the forward movement of VCC will pull the dura forward, keep the dura tension to a certain extent, and form a "tent effect", thus blocking the compression of spinal cord by hematoma. So, the ACAF theoretically completely avoid the risk of compression of spinal cord by hematoma after operation.

\section{Limitations}


Due to its retrospective nature and short-time follow-up, the results of this study might be limited. A more convincing clinical conclusion would be drawn if a larger asymptomatic cohort is included.

\section{Conclusions}

The results of this study showed that ACAF technique can expand spinal canal volume by completing the anterior displacement of the compressive substance in the treatment of MCSMSS. It can safely and effectively relieve the problems of spinal cord compression and spinal canal stenosis, significantly improve cervical curvature and spinal cord morphology. Additionally, ACAF can reduce the rates of complications such as dura tear and hematoma that often occur in traditional anterior operation. In conclusion, ACAF is a satisfactory optional surgical method for the treatment of MCSMSS.

\section{Declarations}

\section{Ethics approval and consent to participate}

All procedures performed in studies involving human participants were in accordance with the ethical standards of the Ethics Committee of Changzheng Hospital.

\section{Consent for publication}

Informed consent was obtained from all individual participants included in the study.

\section{Availability of data and material}

Data sharing is not applicable to this article as no datasets were generated or analysed during the current study.

\section{Competing interests}

The authors declare that they have no competing interests.

\section{Funding}

This study was funded by 
Innovation Fund for College Students of Second Military Medical University (No. FH2017077) by Xi Luo a $\ddagger$

Innovation Fund for College Students of Second Military Medical University (No. MS2016045) by Xi Luo a $\ddagger$

\section{Authors' contributions}

"XL analyzed and interpreted the patient data regarding the MCSMSS, and was a major contributor in writing the manuscript. KS and JS (Jingchuan Sun) performed the examination of the data, and substantively revised the manuscript. SW and YW conducted the acquisition of data. HY have helped draft the work. JJ and YG proposed the ieda of sutdy design. JS (Jiangang Shi) finished the final assessment of the manuscript. All authors read and approved the final manuscript."

\section{Acknowledgements}

Not applicable.

\section{References}

1. Hayashi H, Okada K, Hamada M, Tada K, Ueno R: Etiologic factors of myelopathy. A radiographic evaluation of the aging changes in the cervical spine. Clin Orthop 1987, 214(214):200-209.

2. Melancia JL, Francisco AF, Antunes JL: Spinal stenosis. Handb Clin Neuro/ 2014, 119:541-549.

3. Zhang J, Liu H, Bou EH, Jiang W, Zhou F, He F, Yang H, Liu T: Comparative Study Between Anterior Cervical Discectomy and Fusion with ROI-C Cage and Laminoplasty for Multilevel Cervical Spondylotic Myelopathy without Spinal Stenosis. World Neurosurg 2019, 121:e917-e924.

4. Zhu B, Xu Y, Liu X, Liu Z, Dang G: Anterior approach versus posterior approach for the treatment of multilevel cervical spondylotic myelopathy: a systemic review and meta-analysis. Eur Spine J 2013, 22(7):1583-1593.

5. Li Z, Wang H, Tang J, Ren D, Li L, Hou S, Zhang H, Hou T: Comparison of Three Reconstructive Techniques in the Surgical Management of Patients With Four-Level Cervical Spondylotic Myelopathy. Spine (Phila Pa 1976) 2017, 42(10):E575-e583.

6. Lao L, Zhong G, Li X, Qian L, Liu Z: Laminoplasty versus laminectomy for multi-level cervical spondylotic myelopathy: a systematic review of the literature. J Orthop Surg Res 2013, 8(1):45.

7. Ashkenazi E, Smorgick Y, Rand N, Millgram MA, Mirovsky Y, Floman Y: Anterior decompression combined with corpectomies and discectomies in the management of multilevel cervical myelopathy: a hybrid decompression and fixation technique. J Neurosurg Spine 2005, 3(3):205-209. 
8. Kang Y, Lee JW, Koh YH, Hur S, Kim SJ, Chai JW, Kang HS: New MRI grading system for the cervical canal stenosis. AJR Am J Roentgenol 2011, 197(1):W134-140.

9. Harrison DE, Harrison DD, Cailliet R, Troyanovich SJ, Janik TJ, Holland B: Cobb method or Harrison posterior tangent method: which to choose for lateral cervical radiographic analysis. Spine (Phila Pa 1976) 2000, 25(16):2072-2078.

10. Odate S, Shikata J, Kimura H, Soeda T: Hybrid Decompression and Fixation Technique Versus Plated 3-Vertebra Corpectomy for 4-Segment Cervical Myelopathy: Analysis of 81 Cases With a Minimum 2Year Follow-Up. Clinical spine surgery 2016, 29(6):226-233.

11. Sakai K, Okawa A, Takahashi M, Arai Y, Kawabata S, Enomoto M, Kato T, Hirai T, Shinomiya K: Fiveyear follow-up evaluation of surgical treatment for cervical myelopathy caused by ossification of the posterior longitudinal ligament: a prospective comparative study of anterior decompression and fusion with floating method versus laminoplasty. Spine (Phila Pa 1976) 2012, 37(5):367-376.

12. Yang $H$, Lu X, Wang $X$, Chen D, Yuan W, Yang L, Liu Y: A new method to determine whether ossified posterior longitudinal ligament can be resected completely and safely: spinal canal "Rule of Nine" on axial computed tomography. Eur Spine J 2015, 24(8):1673-1680.

13. Yang H, Lu X, Wang X, Chen D, Yuan W, Yang L, Liu Y: A new method to determine whether ossified posterior longitudinal ligament can be resected completely and safely: spinal canal "Rule of Nine" on axial computed tomography. Eur Spine J 2015, 24(8):1673-1680.

14. Shin JH, Steinmetz MP, Benzel EC, Krishnaney AA: Dorsal versus ventral surgery for cervical ossification of the posterior longitudinal ligament: considerations for approach selection and review of surgical outcomes. Neurosurg Focus 2011, 30(3):E8.

15. Yun JB, Lee JW, Lee E, Jin SY, Kim KJ, Kang HS: Cervical compressive myelopathy: flow analysis of cerebrospinal fluid using phase-contrast magnetic resonance imaging. Eur Spine J 2016, 26(1):1-9.

16. Sun K, Wang S, Sun J, Guo Y, Huan L, Xu X, Sun X, Zhang B, Wang Y, Shi J: Analysis of the Correlation Between Cerebrospinal Fluid Space and Outcomes of Anterior Controllable Antedisplacement and Fusion for Cervical Myelopathy Due to Ossification of the Posterior Longitudinal Ligament. World Neurosurg 2019, 122:e358-e366.

17. Katsumi K, Yamazaki A, Watanabe K, Ohashi M, Shoji H: Can prophylactic Bilateral C4/5 Foraminotomy Prevent Postoperative C5 Palsy After Open. Spine (Phila Pa 1976) 2011, 37(9):748754.

18. Tian Y, Yu KY, Wang YP, Qian J, Qiu GX: MANAGEMENT OF CEREBROSPINAL FLUID LEAKAGE FOLLOWING CERVICAL SPINE SURGERY. Chin Med Sci J 2008, 23(2):121-125.

\section{Tables}


Table 1. Demographics and Clinical Outcomes

\begin{tabular}{|c|c|c|c|}
\hline & HDF & ACAF & $P$ \\
\hline Number of patients & 40 & 45 & \\
\hline \multicolumn{4}{|l|}{ Gender } \\
\hline Male & 22 & 33 & \\
\hline Female & 18 & 12 & \\
\hline Age (years) & $56.1 \pm 9.6$ & $53.0 \pm 10.1$ & 0.465 \\
\hline \multicolumn{4}{|l|}{ Operative level } \\
\hline 3-level & 17 & 20 & \\
\hline 4-level & 23 & 25 & \\
\hline Operation duration (min) & $143.5 \pm 16.1$ & $183.2 \pm 14.0$ & $0.000^{*}$ \\
\hline Blood loss (ml) & $263.3 \pm 19.7$ & $320.7 \pm 17.3$ & $0.000^{*}$ \\
\hline \multicolumn{4}{|l|}{ JOA score } \\
\hline preoperation & $7.2 \pm 2.7$ & $8.3 \pm 4.0$ & 0.378 \\
\hline postoperation & $13.3 \pm 2.3$ & $14.4 \pm 2.6$ & 0.887 \\
\hline \multicolumn{4}{|l|}{ NDI index } \\
\hline preoperation & $17.9 \pm 4.1$ & $18.8 \pm 5.4$ & 0.625 \\
\hline postoperation & $6.2 \pm 3.4$ & $3.3 \pm 0.8$ & 0.155 \\
\hline \multicolumn{4}{|l|}{ Cobb's angle $\left({ }^{\circ}\right)$} \\
\hline preoperation & $15.5 \pm 8.3$ & $14.0 \pm 9.8$ & 0.660 \\
\hline postoperation & $17.9 \pm 4.8$ & $25.3 \pm 5.0$ & $0.000^{*}$ \\
\hline \multicolumn{4}{|l|}{ Complications } \\
\hline CSF leakage & 5 & 1 & \\
\hline Neurologic deterioration & 2 & 0 & \\
\hline Implant complications & 4 & 0 & \\
\hline C5 palsy & 2 & 0 & \\
\hline Dysphagia & 3 & 4 & \\
\hline Epidural hematoma & 2 & 0 & \\
\hline
\end{tabular}

Values are presented as mean $\pm \mathrm{SD}$, or number of patients.

*Statistically significant. 
Table 2. CT measurement

\begin{tabular}{|c|c|c|c|}
\hline & $\mathrm{HDF}$ & ACAF & $\mathbf{P}$ \\
\hline \multicolumn{4}{|l|}{ C3 diameter of spinal canal (mm) } \\
\hline preoperation & $8.5 \pm 1.3$ & $8.9 \pm 1.4$ & 0.062 \\
\hline postoperation & $10.6 \pm 0.9$ & $12.6 \pm 1.8$ & $0.001^{*}$ \\
\hline C3 decompression width (mm) & $15.3 \pm 1.0$ & $17.3 \pm 1.1$ & $0.000^{*}$ \\
\hline \multicolumn{4}{|l|}{ C3 spinal canal area $\left(\mathrm{mm}^{2}\right)$} \\
\hline preoperation & $76.5 \pm 6.8$ & $73.1 \pm 8.2$ & 0.219 \\
\hline postoperation & $119.8 \pm 12.3$ & $139.5 \pm 15.5$ & $0.001^{*}$ \\
\hline \multicolumn{4}{|l|}{ C4 diameter of spinal canal (mm) } \\
\hline preoperation & $9.6 \pm 1.6$ & $8.9 \pm 1.6$ & 0.609 \\
\hline postoperation & $10.2 \pm 1.6$ & $12.6 \pm 3.1$ & $0.011^{*}$ \\
\hline C4 decompression width (mm) & $14.9 \pm 1.1$ & $16.9 \pm 1.1$ & $0.000^{*}$ \\
\hline \multicolumn{4}{|l|}{ C4 spinal canal area $\left(\mathrm{mm}^{2}\right)$} \\
\hline preoperation & $77.6 \pm 9.2$ & $74.8 \pm 9.0$ & 0.406 \\
\hline postoperation & $119.2 \pm 11.3$ & $134.5 \pm 24.2$ & $0.035^{*}$ \\
\hline \multicolumn{4}{|l|}{ C5 diameter of spinal canal (mm) } \\
\hline preoperation & $8.1 \pm 1.3$ & $8.7 \pm 1.8$ & 0.221 \\
\hline postoperation & $11.9 \pm 1.0$ & $15.7 \pm 2.3$ & $0.000^{*}$ \\
\hline C5 decompression width (mm) & $16.1 \pm 1.1$ & $18.1 \pm 1.4$ & $0.000^{*}$ \\
\hline \multicolumn{4}{|l|}{ C5 spinal canal area $\left(\mathrm{mm}^{2}\right)$} \\
\hline preoperation & $76.8 \pm 8.4$ & $76.2 \pm 11.9$ & 0.869 \\
\hline postoperation & $126.7 \pm 6.9$ & $147.1 \pm 23.7$ & $0.003^{*}$ \\
\hline \multicolumn{4}{|l|}{ C6 diameter of spinal canal (mm) } \\
\hline preoperation & $10.3 \pm 1.5$ & $10.7 \pm 1.9$ & 0.358 \\
\hline postoperation & $13.7 \pm 0.9$ & $15.6 \pm 2.1$ & $0.006^{*}$ \\
\hline C6 decompression width (mm) & $16.3 \pm 1.2$ & $18.4 \pm 1.5$ & $0.000^{*}$ \\
\hline \multicolumn{4}{|l|}{ C6 spinal canal area $\left(\mathrm{mm}^{2}\right)$} \\
\hline preoperation & $81.9 \pm 4.5$ & $77.7 \pm 8.3$ & 0.094 \\
\hline postoperation & $134.1 \pm 8.6$ & $147.6 \pm 21.1$ & $0.030^{*}$ \\
\hline
\end{tabular}

Values are presented as mean \pm SD.

*Statistically significant. 


\begin{tabular}{cccc}
\hline & HDF & ACAF & P \\
\hline Grading $\square$ preoperation $\square$ & & & 0.324 \\
\hline 0 & 0 & 0 & \\
\hline 1 & 15 & 15 & \\
2 & 12 & 9 & \\
\hline 3 & 13 & 21 & \\
\hline Grading $\square$ postoperation $\square$ & & & $0.006^{*}$ \\
\hline 0 & 12 & 30 & \\
\hline 1 & 9 & 5 & \\
\hline 2 & 9 & 0 & \\
\hline 3 & 10 & 10 & \\
\hline
\end{tabular}

*Statistically significant

Figures

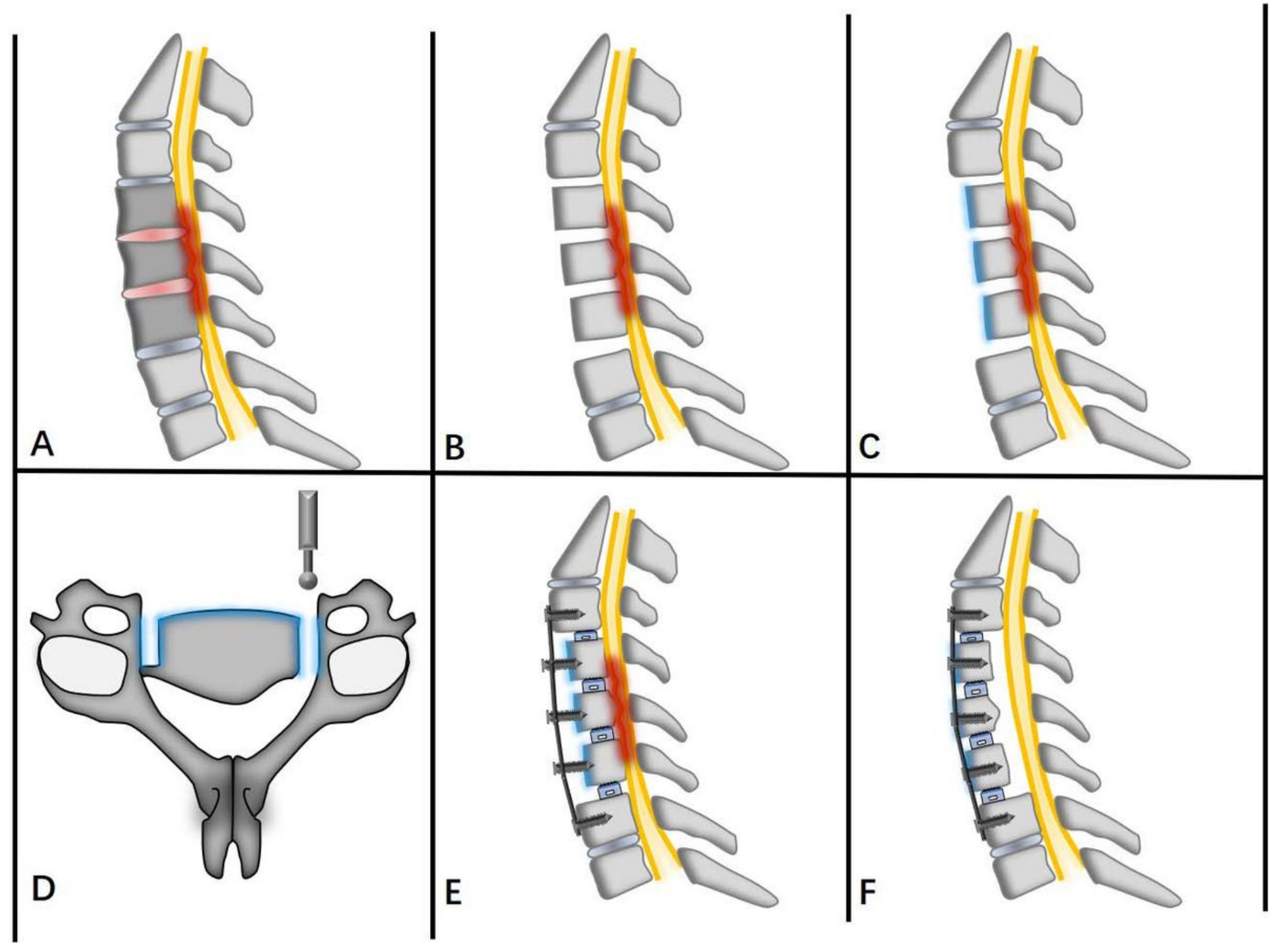




\section{Figure 1}

The procedure of ACAF. (A) Expose responsible vertebral discs and vertebrae. (B) Remove the responsible intervertebral discs and osteophytes of anterior and posterior edge. (C) Perform osteotomy to the anterior parts of vertebrae. (D) Groove at both sides, but retain the posterior wall of the vertebra at one side. (E) Install titanium plate, vertebral screws and intervertebral fusion cages, and resect the posterior wall of the vertebra at the remaining side to make the vertebra free. (F) Hoist the complex of titanium plate, vertebral screws, intervertebral fusion cages and vertebrae.

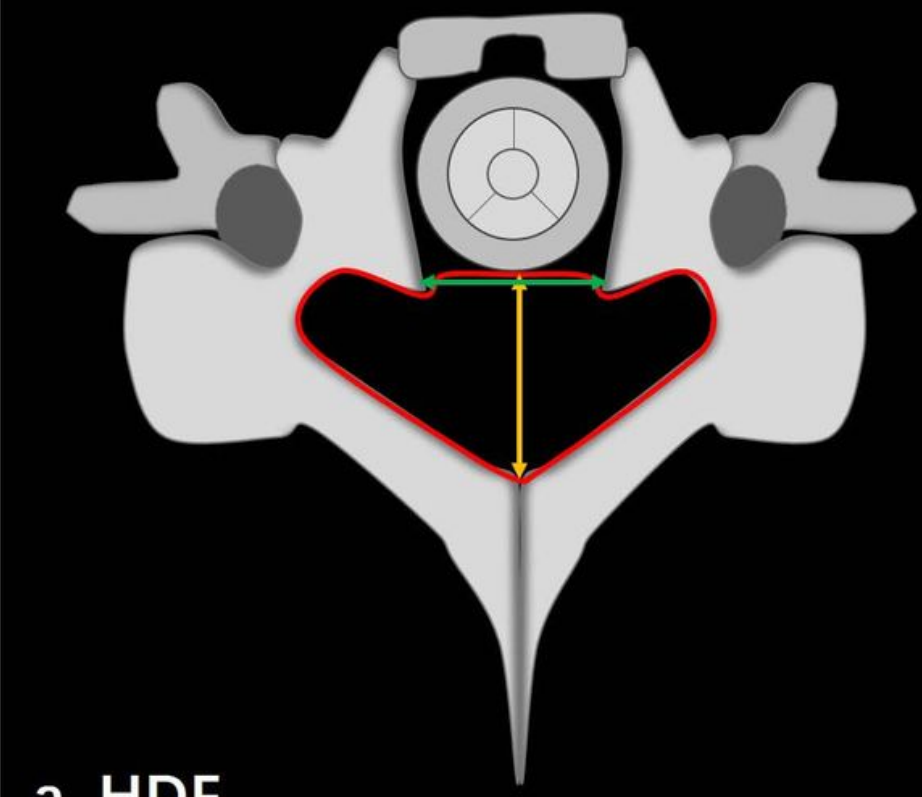

a. HDF

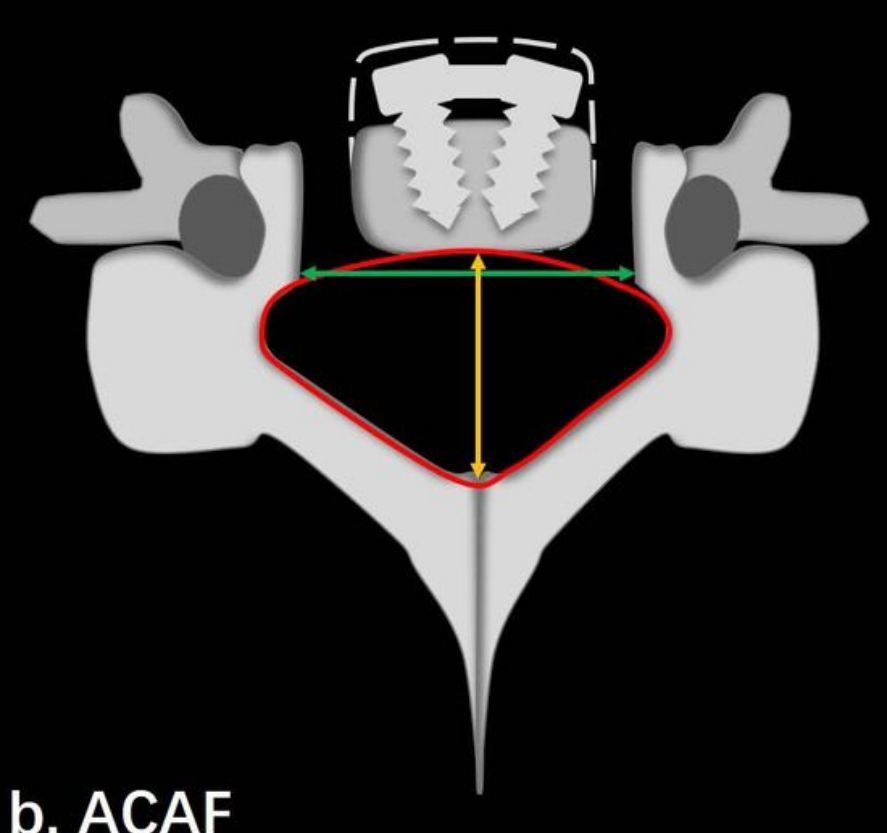

\section{Figure 2}

The measuring diagram on CT axis images of HDF (a) and ACAF (b). The red curve shows the transverse area of decompression. The green line and yellow line represent the decompression width and sagittal diameter of spinal canal, respectively. 

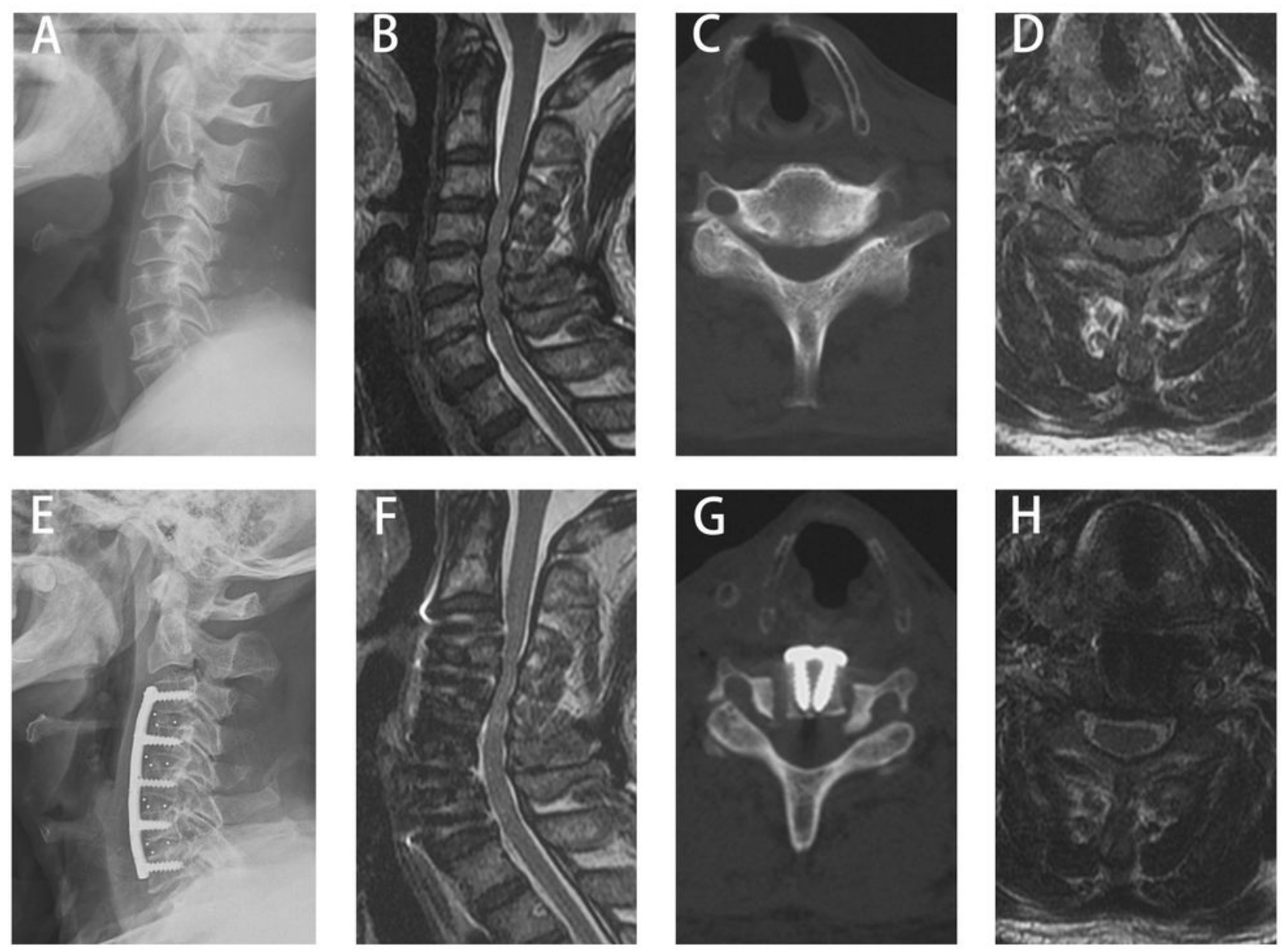

\section{Figure 3}

A 57-year-old man being performed ACAF. (A-D) and (E-H) illustrated preoperative and postoperative conditions, respectively. (A) Lateral X-ray showed the degenerative change and spinal stenosis at C3-5 levels. (B) Lateral MRI demonstrated the compression of CSF band at both discs and vertebrae levels. (C) Axis CT depicted spinal stenosis with spinal canal diameter of $9.6 \mathrm{~mm}$ before surgery. (D) Axis MRI showed no CSF signal anterior to the vertebra. (E) Lateral X-ray revealed the hoisting of C4 and C5 after surgery (F) Lateral MRI confirmed that the CSF band reappear at operating level just after surgery. (G) Axis CT depicted the decompression space with spinal canal diameter of $12.5 \mathrm{~mm}$ after hoisting. $(\mathrm{H})$ Axis MRI showed the normal CSF signal anterior to the vertebra. 

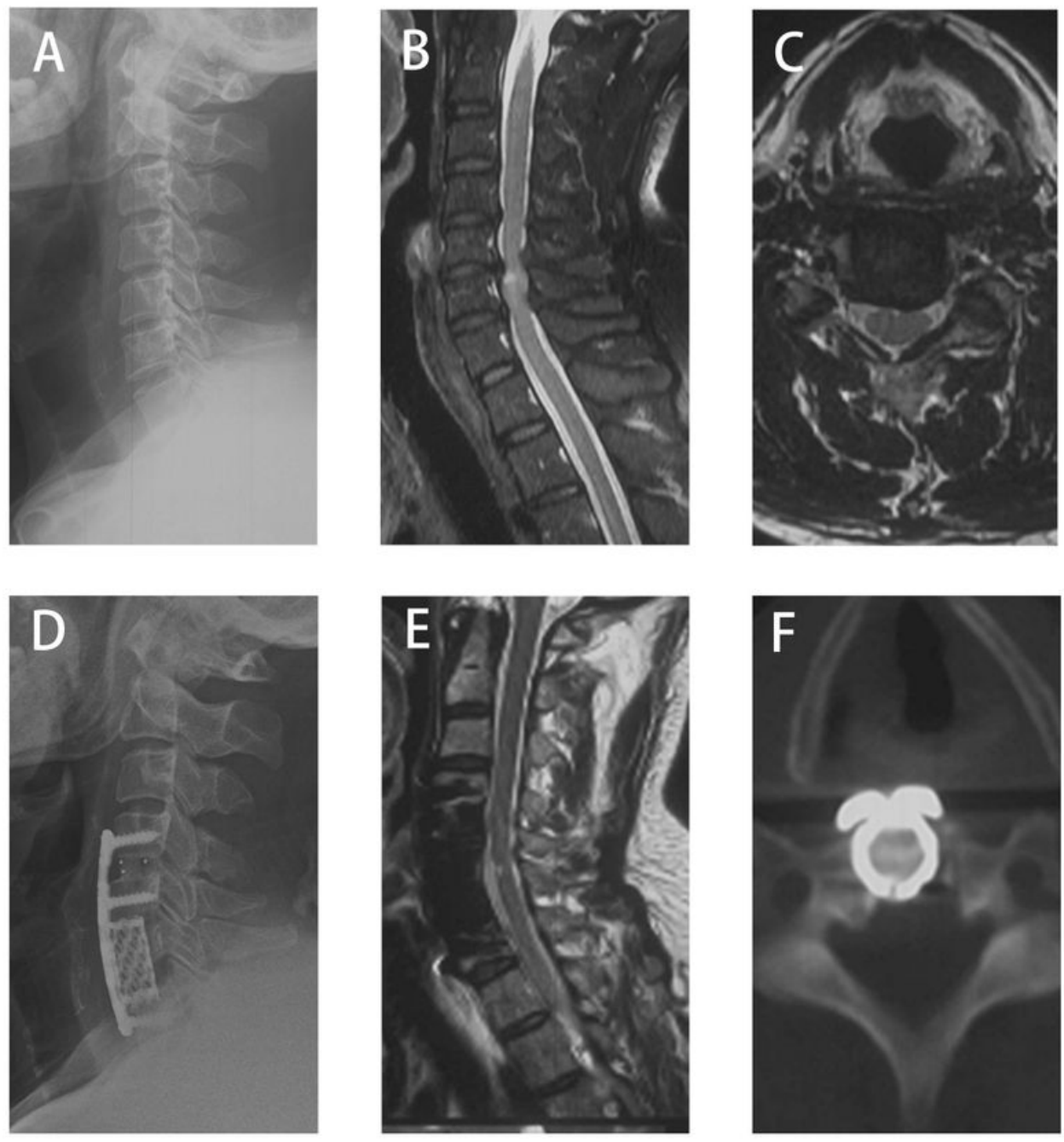

\section{Figure 4}

A-61-year-old man being performed HDF. (A-C) and (D-F) illustrated preoperative and postoperative conditions, respectively. (A) Lateral X-ray showed the degenerative change and spinal stenosis at C4-6 levels. (B) Lateral MRI demonstrated the compression of CSF band at both discs and vertebrae levels. (C) Axis MRI showed CSF signal waned anterior to the vertebra. (D) Lateral X-ray revealed ACDF at C4-5 level and ACCF at C5-6 level. (E) Lateral MRI confirmed that the CSF band reappear at operating level. (F) Axis CT depicted the decompression space after corpectomy and titanium mesh cage implantation. 